\title{
For Propulsion Applications It Is Possible to Utilize Both the Torque Output of the Rotor and the Reactional Torque Acting on the Stator of the Driving Electric Motor by Linearizing the Stator
}

\author{
Salah Abunaieb \\ Sogex Oman LLC, Muscat, Sultanate of Oman \\ Email: salnayib@hotmail.com
}

How to cite this paper: Abunaieb, S. (2021) For Propulsion Applications It Is Possible to Utilize Both the Torque Output of the Rotor and the Reactional Torque Acting on the Stator of the Driving Electric Motor by Linearizing the Stator. Energy and Power Engineering, 13, 163-173. https://doi.org/10.4236/epe.2021.135011

Received: April 17, 2021

Accepted: May 28, 2021

Published: May 31, 2021

Copyright ( 2021 by author(s) and Scientific Research Publishing Inc. This work is licensed under the Creative Commons Attribution International License (CC BY 4.0).

http://creativecommons.org/licenses/by/4.0/

\section{(c) (i) Open Access}

\begin{abstract}
This paper presents a novel idea of utilizing the reactional torque of the conventional electric motor as a linear output for propulsion in addition to the conventional torque output of the rotor. The idea is demonstrated by a theoretical proposal of linearizing the stator of one of the most used motors in Electrical Vehicles and Hybrid Vehicles. The proposed Linear Stator Motor is a simple modification without involving any functional change of the conventional motor. Though theoretical, the indicated possible input energy saving of more than $75 \%$ as compared to the conventional motor is no surprise, as by linearizing the stator, an almost equal linear propulsion output is added to the conventional rotor output. In addition to this remarkable saving in input energy, the proposed Linear Stator Motor that suits all types of vehicles, can maintain propulsion without the need for a mechanical transmission system. Also, in the case of watercraft and aircraft vehicles, no external mechanical propulsion drive system is required. It is just an internal force that can push the vehicle forward, backward, or laterally, while the conventional rotor output can be utilized for energy recovery by driving a DC generator.
\end{abstract}

\section{Keywords}

BLDC Motor, Electrical Vehicle, Hall Effect, Linearization, Newton's Third Law of Motion, Propulsion, Rotor, Stator

\section{Introduction}

Newton's Third law of Motion states that when one object exerts a force on a 
second object, the second object simultaneously exerts a force equal in magnitude and opposite in direction on the first object [1]. On the other hand, work is defined as the product of force and displacement. So, a force is said to do positive work if (when applied) it has a component in the direction of the displacement of the point of application [2]. In the conventional type of electric motors considered in this study, the interaction between the magnetic field of the rotor and the electromagnetic field of the stator, makes the rotor and the stator exert equal and opposite forces on each other. The force acting on the rotor produces angular movement which is the useful output work or torque that drives the connected load. While the force acting on the stator does not do any work as there is no resulting displacement. The stator of the conventional motor is a closed loop that is, in the case of propulsion applications, fixed to the mechanically balanced body of the vehicle. So, by opening this loop, i.e., by Linearizing the stator, the reactional force acting on it can be utilized as a linear propulsion force that can push the vehicle forward, backward, or laterally. This, while the rotor output can be utilized the same way as in conventional electric vehicles or otherwise in driving a generator to recover part of the electric energy required to run the motor. The idea is demonstrated by a theoretical proposal of linearizing the stator of the conventional Brushless DC (BLDC) motor. The proposed Linearization of the stator, as will be seen in the following sections, does not involve any functional change of the conventional BLDC motor. The principle of work, the electrical connections, the angular rotor position detectors and the commutation system are all maintained the same as in the conventional BLDC motor. For the option of utilizing the resulting linear stator force for propulsion and the rotor output for driving a DC generator, more than $75 \%$ of the power as compared to the conventional BLDC motor, can be saved.

\section{Brief Description of the Conventional BLDC Motor}

The BLDC motor shown in Figure 1, is one of the most used motors in Electrical Vehicles and Hybrid Vehicles. In this motor, the rotor is fitted with permanent magnets and the stator with windings around each of the stator poles.

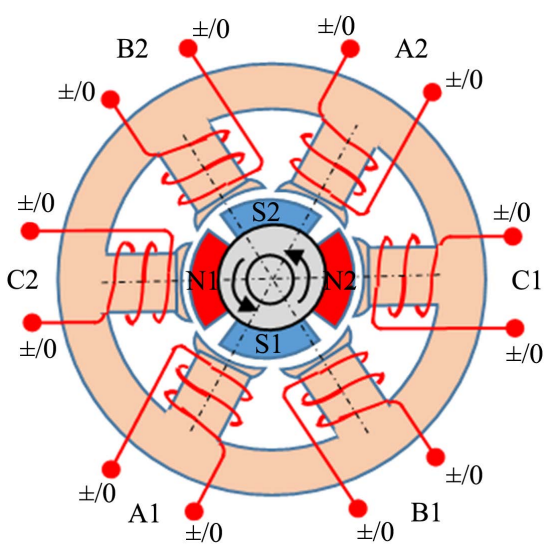

Figure 1. Conventional BLDC motor. 
When electric power is passing through the windings it sets electromagnetic field that interacts with the permanent magnets to maintain the rotation of the rotor. The power to this motor is a three-phase DC supply that is commutated electronically to energize the stator windings in a sequence that follows the angular position of the rotor. The angular position of the rotor is commonly detected using three Hall detectors or a rotating encoder and the position signal is fed back to the sequence control circuit. Every two opposite poles of the total six stator poles are connected as one pole to one of the three phases A, B, or C [3].

\section{Linearizing the Stator of the Conventional BLDC Motor}

Now, to linearize the stator of the conventional BLDC motor, the stator poles are first marked with the numbers 1 to 6 in the direction of rotation and the stator ring is marked for cutting into three segments with each segment consisting of two stator poles as shown in Figure 2(a).

After cutting, then counter to the direction of rotation, Segment 2 is rotated by $120^{\circ}$ and placed behind Segment 1 , and Segment 3 by $240^{\circ}$ and placed behind Segment 2. Like that, the three segments are put one behind the other in a line (Line of Segments) as shown in Figure 2(b).

The rotor is axially extended to accommodate three Permanent Magnet Sets (PM Set 1, $2 \& 3$ ) as shown in Figure 3(a) and is mounted above the stator segments with the air gap adjusted same as in the intact motor.

The three PM Sets are fitted to the rotor at relative angular positions so that each PM Set faces the corresponding Stator Segment (Figure 3(b)) the same way as in the intact motor (Figure 2(a)). So, same as the corresponding Stator Segments, PM Set 2 and PM Set 3 are mounted and fixed at angular positions shifted relative to PM Set 1 respectively by $120^{\circ}$ and $240^{\circ}$ opposite to the direction of rotation.

The rotor angular position detectors, e.g., three Hall Effect detectors, are placed same way as in the in the conventional motor to detect only one PM Set

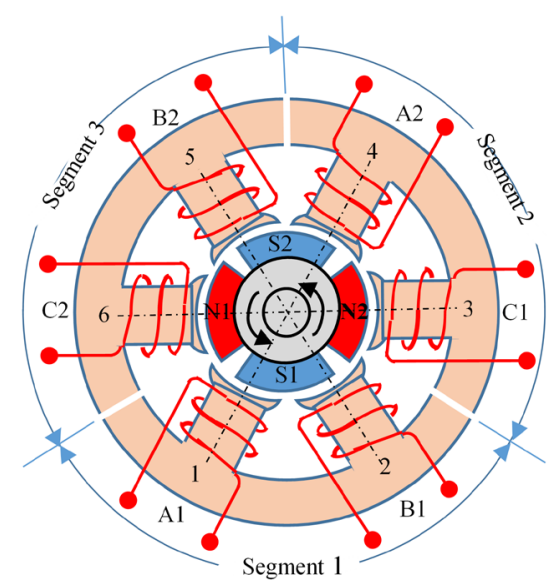

(a)

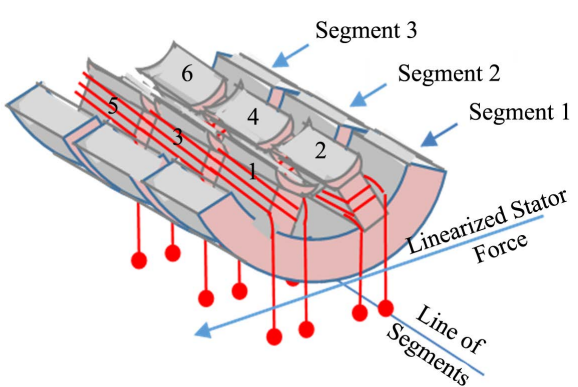

(b)

Figure 2. (a) Marking the stator for cutting into three segment; (b) The stator three segment placed one behind the other. 


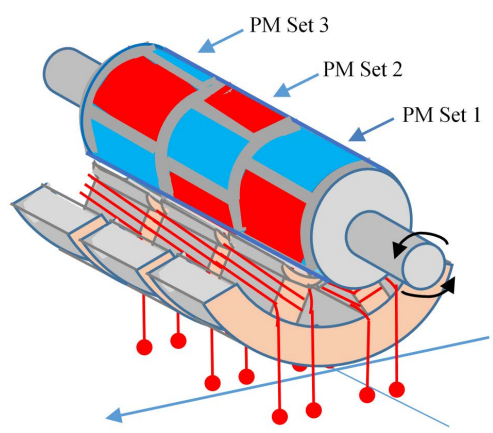

(a)
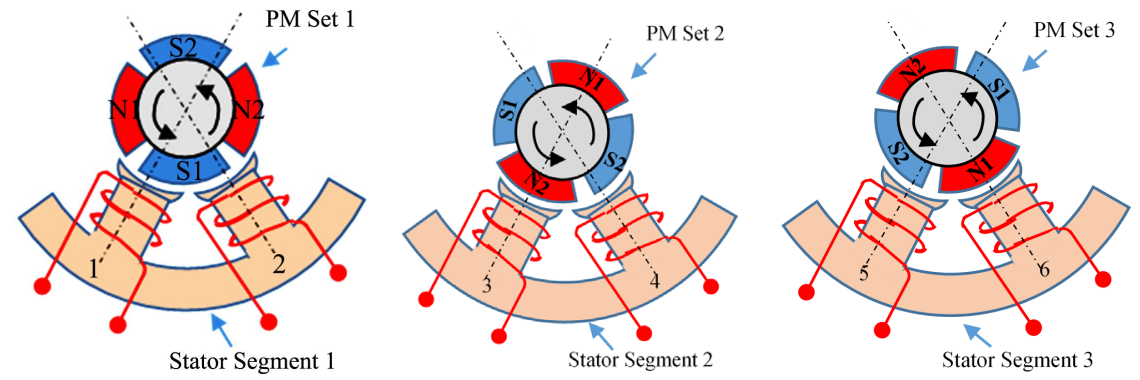

(b)

Figure 3. (a) The axially extended rotor mounted above the stator segments; (b) The three stator segments and the position of the corresponding PM sets.

(PM Set 1, say). The other two PM sets, as explained above, are of fixed relative angular positions with respect to the detected PM set. The power supply connections, the electronic circuits and the commutation sequence remain unchanged. So, in the same sequence of commutation the interaction between the electromagnetic field of the stator poles and the rotor magnets maintains rotation of the rotor of this theoretical Linear Stator (LS) Motor and in the same direction of rotation as in the conventional BLDC motor.

The rotor is aligned so that its axis is parallel to the line of segments. The line of segments as shown in Figure 2(b) is the intersection between the plane on which the stator segments are sitting and the axial plane of symmetry of the stator segments. The axis of the rotor and the line of segments both lie on the axial plane of symmetry of the stator segments. In this way the linearized stator output is always perpendicular to the axial plane of symmetry. The stator segments and the bearing housings of the rotor are fixed to a supporting frame which in turn is fixed to the chassis of the vehicle. An alternative way is to pivot the supporting frame to a secondary frame that in turn is pivoted to the chassis. This latter alternative allows to mechanically change the direction of the linearized stator output around two perpendicular axes relative to the chassis. So, while the rotor is kept running at an optimum speed and torque, the magnitude of the component of the linearized force in the direction of the forward or reverse displacement can be changed by tilting the supporting frame around the horizontal axis, while steering and lateral movement can also be controlled by turning the secondary frame around the vertical axis. 


\section{The Stator Linearized Force and Its Utilization}

As shown in Figure 4, the stator electromagnetic field interaction with the permanent magnets of the rotor creates two equal and opposite forces between the rotor and each of the stator poles. One is $\mathrm{F}_{\mathrm{r}}$ that acts tangentially on the rotor to maintain the rotational/torque output. The other is $F_{s}$ that acts on the stator which is fixed to the chassis of the vehicle.

The force $\mathrm{F}_{\mathrm{s}}$ acting on the left and that acting on the right-hand side of the stator can each be analysed into two vector components. One component $\left(\mathrm{F}_{\mathrm{s}}\right.$ $\sin 30)$ is perpendicular to the line of movement of the vehicle and the other $\left(\mathrm{F}_{\mathrm{s}}\right.$ $\cos 30$ ) is parallel to the line of movement of the vehicle. As shown in Figure 4, the perpendicular vector components $\left(\mathrm{F}_{\mathrm{s}} \sin 30\right)$ on the two sides of the stator are equal in magnitude and opposite in direction forming a couple with a perpendicular distance of around the radius of the rotor between the two forces. The resultant moment/reactional torque of this couple is applied on the chassis and its effect is counteracted the same way the full reactional torque is counteracted in conventional motor driven vehicles. While in parallel to the line of movement of the vehicle, the left- and right-hand side components $\left(\mathrm{F}_{\mathrm{s}} \cos 30\right)$ are in the same direction and hence they add to maintain the proposed Stator Propulsion Force which is the main objective of this study.

In the above considered modification the stator is not $100 \%$ linear. The factor cos30, which is equal to 0.866 , limits linearity to $86.6 \%$ and consequently limits the total average Stator Linear Output Force in magnitude to $86.6 \%$ of the total average force acting on the stator or that acting on the rotor. This is because of the choice of cutting the stator ring into only three segments, each consisting of two stator poles making a central angle of $60^{\circ}$. So, each side of the stator is inclined by $30^{\circ}$ with respect to the vertical and hence comes the factor cos 30 . Of course, it is possible to make it very close to $100 \%$ linear by cutting the stator ring into six segments each consisting of only one stator pole with a small central angle corresponding to the arc length of the stator pole. The problem with this latter choice is that the rotor will then need to be extended by six times and fitted with six PM sets. So, the choice of sacrificing around $13 \%$ of linearity is believed

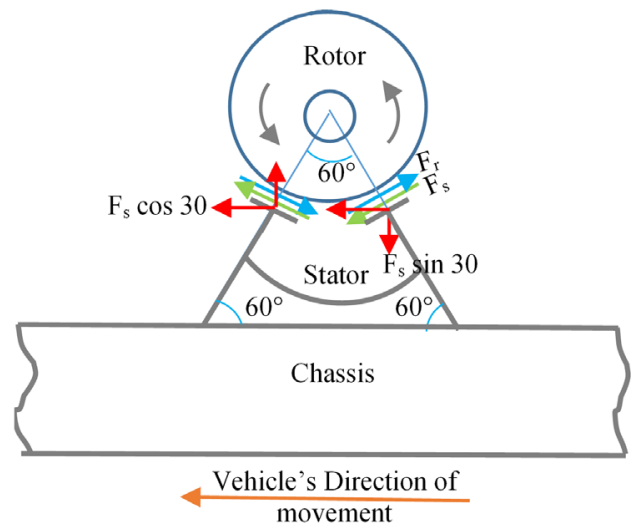

Figure 4. Linear stator force analysis. 
to be justified by optimizing the length of the rotor and the number of PM sets. It will be seen later that the gap between the $86.6 \%$ and $100 \%$ linearity is even much narrower when comparing the resulting saving of energy/power in each case.

Now we have two outputs, one is the conventional torque output of the rotor and the other is the linear output of the stator. Well, it must be noted here that the stator linearized output is dependent on the rotor output. In other words, there will be output from the stator if and only if the rotor is driving some non-zero output load. The two outputs can both be utilized in propulsion applications in one of two options as below:

Option 1: Utilizing both outputs for propulsion of the vehicle as shown below in Figure 5(a).

Option 2: Utilizing the linear output of the stator for propulsion, and the torque output of the rotor for driving a DC generator to recover part of the energy required to drive the motor as shown in Figure 5(b).

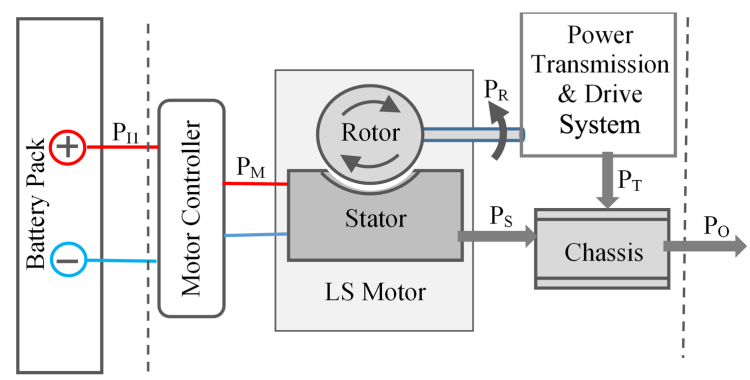

(a)

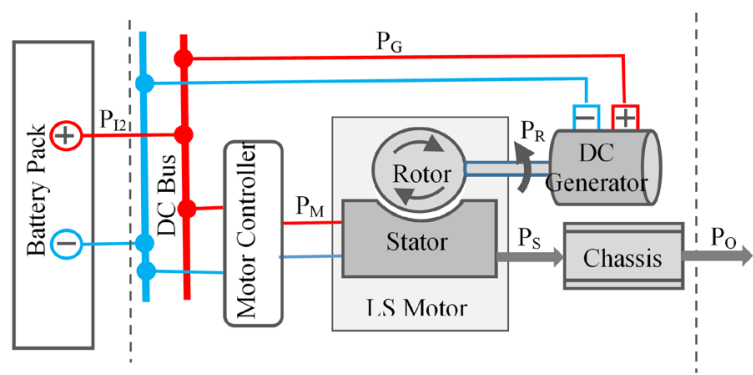

(b)

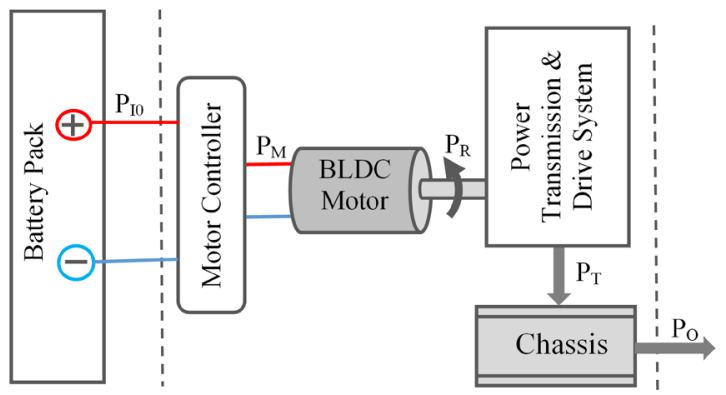

(c)

Figure 5. (a) LS motor option 1 driven vehicle; (b) LS motor Option 2 driven vehicle; (c) Conventional BLDC motor driven vehicle. 
For a given output power $\mathrm{P}_{\mathrm{O}}$, the input power $\mathrm{P}_{\mathrm{I}}$ required in each of the above two options is compared to the input power required for the conventional BLDC motor shown in Figure 5(c) to maintain the same output power.

\section{LS Motor (Options 1 \& 2) Required Input Power vs. the BLDC Motor to Maintain the Same Output}

The following simple assumptions, provisions and formulae are used to compare the input power requirement of the two LS motor utilization options to that required by the conventional BLDC motor to maintain the same propulsion output.

Assumptions and provisions:

- $\eta_{M} \equiv$ the efficiency of the LS motor which is assumed to be equal to that of the BLDC motor. Based on typical BLDC motor efficiency range of $85 \%$ to $90 \%[4], \eta_{M}=85 \%$

- $\eta_{T} \equiv$ Mechanical Transmission System efficiency. Considering a typical Transmission efficiencies range of $86 \%$ to $98 \%$ [5], $\eta_{T}=92 \%$.

- $\eta_{G} \equiv$ DC generator efficiency. Considering a typical efficiencies range of $85 \%$ to $95 \%[6], \eta_{G}=90 \%$.

- $\mu \equiv$ linearity factor. $\mu=0.866 @ 86.6 \%$ linear stator, and = $1 @ 100 \%$ linear stator.

- $P_{O} \equiv$ the output power required to maintain movement of the vehicle at some given speed. $P_{O}=1$ unit of power.

- $P_{10} \equiv$ BLDC motor input power provided from the battery.

- $P_{I \mathrm{l}} \equiv$ Option 1 LS motor input power provided from the battery.

- $P_{R} \equiv$ Option 2 LS motor input power provided from the battery.

- $P_{M} \equiv$ the controlled power input to the motor in each case.

- $P_{R} \equiv$ the torque output of the rotor in each case.

- $P_{S} \equiv$ the linear stator output of the LS motor in each of the two options.

- $P_{G} \equiv$ the Dc generator output.

- $P_{T} \equiv$ Mechanical Transmission \& Drive System output.

- $S P \equiv$ Saved Power which is equal to the difference between the BLDC power input $P_{I 0}$ and the LS motor input $P_{I}$ in case of Option 1 and $P_{R}$ in case of Option 2.

Formulae:

1) Conventional BLDC motor (Figure 5(c)):

$$
\begin{gathered}
P_{T}=P_{O}=1 \\
P_{R}=P_{T} / \eta_{T}=1 / \eta_{T} \\
P_{M}=P_{R} / \eta_{M}=1 / \eta_{T} / \eta_{M} \\
P_{I 0}=P_{M}=1 / \eta_{T} / \eta_{M}
\end{gathered}
$$

2) LS motor Option 1 (Figure 5(a)):

$$
\begin{gathered}
P_{O}=P_{T}+P_{S}=1 \\
P_{T}=\eta_{T} * P_{R}
\end{gathered}
$$




$$
P_{S}=\mu * P_{R}
$$

From (5), (6) and (7):

$$
\begin{gathered}
P_{R}=P_{O} /\left(\mu+\eta_{T}\right)=1 /\left(\mu+\eta_{T}\right) \\
P_{M}=P_{R} / \eta_{M}=1 /\left(\mu+\eta_{T}\right) / \eta_{M} \\
P_{I 1}=P_{M}=1 /\left(\mu+\eta_{T}\right) / \eta_{M} \\
S P=P_{I 0}-P_{I 1} \\
S P \%=\left(\left(P_{I 0}-P_{I 1}\right) / P_{I 0}\right) \%
\end{gathered}
$$

3) LS motor Option 2 (Figure 5(b)):

$$
\begin{gathered}
P_{S}=P_{O}=1 \\
P_{R}=P_{S} / \mu=1 / \mu \\
P_{G}=\eta_{G} * P_{R}=\eta_{G} / \mu \\
P_{M}=P_{R} / \eta_{M}=1 / \mu / \eta_{M} \\
P_{I 2}=P_{M}-P_{G}=\left(1 / \eta_{M}-\eta_{G}\right) / \mu \\
S P=P_{I 0}-P_{I 2} \\
S P \%=\left(\left(P_{I 0}-P_{I 2}\right) / P_{I 0}\right) \%
\end{gathered}
$$

\begin{tabular}{|c|c|c|c|c|c|c|c|c|c|c|c|}
\hline & $P_{O}$ & $P_{R}$ & $P_{S}$ & $P_{T}$ & $P_{G}$ & $P_{M}$ & $P_{I O}$ & $P_{I 1}$ & $P_{I 2}$ & $S P$ & $S P \%$ \\
\hline $\begin{array}{l}\text { Conventional } \\
\text { BLDC Motor }\end{array}$ & 1 & 1.09 & - & 1 & - & 1.28 & 1.28 & - & - & - & - \\
\hline \multicolumn{12}{|c|}{ 86.6\% LINEAR STATOR } \\
\hline LS Motor Option 1 & 1 & 0.56 & 0.48 & 0.52 & - & 0.66 & - & 0.66 & - & 0.62 & $48 \%$ \\
\hline LS Motor Option 2 & 1 & 1.15 & 1 & - & 1.04 & 1.36 & - & - & 0.32 & 0.96 & $75 \%$ \\
\hline \multicolumn{12}{|c|}{ 100\% LINEAR STATOR } \\
\hline LS Motor Option 1 & 1 & 0.52 & 0.52 & 0.57 & - & 0.61 & - & 0.61 & - & 0.67 & $52 \%$ \\
\hline LS Motor Option 2 & 1 & 1 & 1 & - & 0.90 & 1.18 & - & - & 0.28 & 1 & $78 \%$ \\
\hline \multicolumn{12}{|c|}{ Constants } \\
\hline$\eta_{\mathrm{M}}$ & & $\eta_{T}$ & \multicolumn{5}{|c|}{$\begin{array}{c}\mu \text { at } 86.6 \% \\
\text { Linearty }\end{array}$} & \multicolumn{4}{|c|}{$\begin{array}{c}\mu \text { at } 100 \% \\
\text { Linearty }\end{array}$} \\
\hline 0.85 & & 0.92 & & 0.90 & & \multicolumn{2}{|c|}{0.87} & \multicolumn{4}{|c|}{1} \\
\hline
\end{tabular}

The results of above formulae and assumptions are shown in Table 1 below.

\section{Discussion}

As shown above in Table 1, the input power required for either of the two LS motor utilization options, is by far lower than that required for the conventional BLDC motor to maintain the same output power. Consequently, the LS motor Saved Power $(S P)$ is strikingly high. In percentage, $S P$ is $48 \%$ for option 1 and

Table 1. LS motor Options $1 \& 2$ Energy requirements vs BLDC motor. 
$75 \%$ for option 2, at $86.6 \%$ LS motor linearity. At $100 \%$ linearity these values rise slightly to $52 \%$ and $78 \%$ respectively. Still, what is puzzling about the above results is not that the input power required for the LS motor is less than that for the conventional BLDC motor, but that it is less even than the output power. So, for maintaining output power $\left(P_{O}\right)$ equal to 1 unit of power, the $86.6 \%$ linear LS motor option 1 input power $\left(P_{I}\right)$ is 0.66 and that of option $2\left(P_{R}\right)$ is 0.32 only. Yet and unlike it appears, the Law of Conservation of Energy is not violated. A well-known statement of the Law of Conservation of Energy is that energy can neither be created nor destroyed, although it can be transformed from one form to another form of energy [7] [8]. In this proposal, of course, no energy is created. It is the reactional torque that, as per Newton's Third law of Motion, acts counter to the useful rotor output torque. In conventional motor driven vehicles, the unwanted effect of the reactional torque is counteracted in a way or another. An example of counteracting the reactional torque in vehicles is the tail rotor of the helicopter. The tail rotor provides a thrust force that counteracts the reactional torque generated by the spinning of the main rotor [9]. Without this tail rotor the body of the helicopter would spin around its axis in opposite direction to the main rotor. Another function of the tail rotor is some sort of utilization of the reactional torque and the counteracting thrust, as by changing the pitch angle of the blades, the pilot can steer the helicopter [10].

Now, the dilemma is in how to find the efficiency of the LS motor. Following the present methods, the efficiency of the motor is the ratio of the rotor output to the input of the motor. But, the input, which is electrical, is equal to the mechanical rotor output plus the losses which include friction, windage and electrical losses [11]. This implies that in reality, the output of the electric motor is always less than the input and hence the efficiency of the motor is always less than the ideal $100 \%$ efficiency. In conventional motors, the reactional torque, which is equal in magnitude to the rotor output torque, is neither considered as an output nor as a loss. As shown above, by utilizing the reactional torque as an additional propulsion output, the total output of the proposed LS motor is greater than the input, yielding an efficiency of more than $100 \%$ which is not acceptable. However, and at this stage, whether the dilemma lies in the definition of the efficiency of the motor or in equivalence of electrical and mechanical energy, is all left to remain out of the scope of this paper.

Further to above, Table 1 provides energy and power capacity comparison between the LS motor utilization option 1 and option 2 . In option 1 , as defined above, the total propulsion output is the sum of that from the rotor through the transmission system and that from the linearized reactional torque acting on the stator. As these two outputs are almost equal the saved power $(S P)$, as can be seen in Table 1 , is around $50 \%$ only. Consequently, the motor size, as indicated by the controlled power input $\left(P_{M}\right)$, is around half of that of the conventional BLDC motor for maintaining the same propulsion output. In option 2 , the propulsion output is the linearized reactional torque acting on the stator, while the rotor output is utilized for energy recovery by driving a DC generator. As 
Table 2. LS motor Option 1 vs. Option 2.

\begin{tabular}{|c|c|c|}
\hline Features & Option 1, LS motor & Option 2, LS motor \\
\hline $\begin{array}{l}\text { Saved Power (SP) as } \\
\text { compared to BLDC motor }\end{array}$ & $48 \%-52 \%$ & $75 \%-78 \%$ \\
\hline $\begin{array}{l}\text { Speed and Torque } \\
\text { of the motor. }\end{array}$ & $\begin{array}{l}\text { Varies with the speed } \\
\text { of the vehicle. }\end{array}$ & $\begin{array}{l}\text { Independent of the speed of } \\
\text { the vehicle, i.e., can run at } \\
\text { optimum speed and torque. }\end{array}$ \\
\hline $\begin{array}{l}\text { Motor capacity compared } \\
\text { to BLDC motor }\end{array}$ & Around half & Around same \\
\hline $\begin{array}{l}\text { Wheels for wheeled and } \\
\text { tracked vehicles }\end{array}$ & $\begin{array}{l}\text { Coupled to the motor either } \\
\text { directly or via transmission system. }\end{array}$ & Not coupled to the motor. \\
\hline $\begin{array}{l}\text { Propeller for air- } \\
\text { and watercraft vehicles }\end{array}$ & Required. & Not required. \\
\hline Gear & Optional & Not required \\
\hline Transmission system & Required in most cases & Not required \\
\hline Generator & Not required & Required \\
\hline For wheeled vehicles & Suitable & Suitable \\
\hline For tracked vehicles & Suitable & Suitable \\
\hline For railed vehicles & Suitable & Suitable \\
\hline For skied vehicles & Suitable & Suitable \\
\hline For watercraft vehicles & Suitable & Suitable \\
\hline For aircraft vehicles & Suitable. & Suitable \\
\hline For space vehicles & Not suitable & Suitable. \\
\hline To fit into existing vehicles & Possible with little modifications. & Possible with little modifications \\
\hline
\end{tabular}

mentioned earlier, the linear stator output is dependent on the rotor output. So, by considering the linearity factor $(\mu)$ of $\leq 1$, the rotor torque output $\left(P_{R}\right)$ must be equal or slightly greater than the stator output $\left(P_{S}\right)$. The rotor output $P_{R}$ is driving the DC generator that generates the recovery power $\left(P_{G}\right)$. Hence, there is more room for energy recovery with option 2 as indicated by $S P$ of up to more than $75 \%$ as shown in Table 1 . The motor in option 2 is around the same size as that of the conventional BLDC motor for maintaining the same propulsion output.

Including other aspects, the comparison between LS motor option 1 and option 2 is summarized below in Table 2 .

\section{Conclusion}

Linearizing the reactional torque of the conventional motor and utilizing it as a propulsion force is possible as simply explained by the proposal of modifying the conventional BLDC motor to a Linear Stator (LS) motor. When both the linearized stator output and the conventional rotor output are used for propulsion (Option 1), the required input power of the LS motor is around $50 \%$ of that required for the conventional BLDC motor to maintain the same propulsion out- 
put. While, when utilizing only the stator linearized output for propulsion and the rotor output for driving a DC generator to recover energy (Option 2), the required power is around $25 \%$ of that required for the conventional BLDC motor. Though theoretical, the above results are not far from reasonable when we bear in mind the conclusive idea that by linearizing the stator, an almost equal output is added to the conventional rotor propulsion output. The merits of the LS motor utilization Option 2 over Option 1, are not only limited to more energy saving. In Option 2 the linear stator output is simply a force that is directly pushing the vehicle without any need for a transmission system and without a propeller for watercraft and aircraft vehicles. It may also be suitable for spacecraft where it can propel without shooting out fuel. Though the proposal is based on the modification of the BLDC motor and without any functional change, still practical study is required to arrive at an experimental energy requirement of the theoretical LS motor and any other unforeseen practical limitations in comparison to the conventional motors in use for propulsion.

\section{Conflicts of Interest}

The author declares no conflicts of interest regarding the publication of this paper.

\section{References}

[1] Newton's Laws of Motion. Wikipedia. https://en.wikipedia.org/wiki/Newton\%27s_laws_of_motion.

[2] Work (Physics). Wikipedia. https://en.wikipedia.org/wiki/Work_(physics).

[3] Design Guide on DC Motors. Sponsored by Maxon. https://www.motioncontroltips.com/designguide-dc-motors.

[4] Electric Motor. Wikipedia. https://en.wikipedia.org/wiki/Electric_motor\#Brushless_DC_motor.

[5] Břoušek, J. and Zvolský, T. (2018) Experimental Study of Electric Vehicle Gearbox Efficiency. MATEC Web of Conferences, 234, Article No. 02004. https://doi.org/10.1051/matecconf/201823402004 https://doi.org/10.1051/matecconf/2018234004.

[6] DC Generators: Gensets that are Very Reliable with Efficiency Ratings of 85-95\%. Economic Times.

https://economictimes.indiatimes.com/small-biz/productline/power-generation/dcgenerators-gensets-that-are-very-reliable-with-efficiency-ratings-of-85-95/articleshow/690 54755.cms.

[7] Law of Conservation of Energy. https://byjus.com/physics/law-of-conservation-of-energy/.

[8] Conservation of Energy. https://en.wikipedia.org/wiki/Conservation_of_energy.

[9] Tail Rotor. https://en.wikipedia.org/wiki/Tail_rotor.

[10] Tail Rotor Airfoils Stabilize Helicopters, Reduce Noise. (2010) Originating Technology/NASA Contribution. https://spinoff.nasa.gov/Spinoff2010/t_2.html.

[11] Efficiency of DC Motor and DC Generator. Electrical Academia. https://psaimoodriy.com/?l=9FO1QgTGUxQxcxG\&s=256078835286549253\&z=254 28698viewability_action_id=256078835286549253. 